\title{
The Effect of Sagittal Knee Deformity on Preoperative Measurement of Coronal Mechanical Alignment during Total Knee Arthroplasty
}

\author{
Gautam M Shetty, MS (Orth) ${ }^{1}$, Arun Mullaji, FRCS, MCh, MS (Orth) ${ }^{1}$, Ahmed Adel Khalifa, MSc (Orth) $)^{1,2}$, \\ Abhik Ray, MS (Orth) ${ }^{1}$, and Vivek Nikumbha, MS (Orth) ${ }^{1}$ \\ ${ }^{1}$ Department of Orthopaedic Surgery, Breach Candy Hospital \& Mullaji Knee Clinic, Mumbai, India; ${ }^{2}$ Department of Orthopaedic Surgery, Qena University Hospital, \\ Qena, Egypt
}

\begin{abstract}
Purpose: The purpose of this prospective study was to determine correlation between coronal mechanical alignment measured on preoperative standing full-length radiographs and navigation during total knee arthroplasty (TKA) in the presence of an associated sagittal deformity (hyperextension and flexion deformity).

Materials and Methods: Coronal mechanical alignment measured on preoperative, standing, full-length, hip-to-ankle anteroposterior radiographs was compared with intraoperative measurements recorded with computer navigation in 200 primary navigated TKAs.

Results: The mean difference in mechanical alignment between the two techniques was significantly greater ( $\mathrm{p}=0.001)$ in patients with an associated flexion deformity $>10^{\circ}$ when compared to knees with associated flexion deformity $\leq 10^{\circ}$; $48 \%$ of knees with a flexion deformity $>10^{\circ}$ had a difference of $\geq 3^{\circ}$ between the full-length radiograph and navigation alignment measurements. There was a strong correlation between the radiographic and navigation measurement techniques.

Conclusions: The mean difference in coronal mechanical alignment between the two techniques was significantly higher in patients with an associated flexion deformity $>10^{\circ}$. Hence, surgeons should take caution when relying on preoperative full-length radiographs for determining coronal mechanical alignment in patients with an associated flexion deformity $>10^{\circ}$ where using navigation may be more reliable.
\end{abstract}

Keywords: Knee, Arthroplasty, Computer-assisted surgery, Sagittal deformity, Alignment

\section{Introduction}

Assessing coronal alignment, in order to determine the amount of knee deformity preoperatively and during surgery, is an important part of total knee arthroplasty (TKA). Preoperatively, the degree of knee deformity in terms of coronal mechanical align-

Received January 15, 2017; Revised February 24, 2017;

Accepted March 13, 2017

Correspondence to: Gautam M Shetty, MS (Orth)

Department of Orthopaedic Surgery, Breach Candy Hospital \& Mullaji

Knee Clinic, 101, Cornelian, Kemps Corner, Cumballa Hill, Mumbai 400036, India

Tel: +91-22-23856161, Fax: +91-22-23856161

E-mail: gautams10@gmail.com

This is an Open Access article distributed under the terms of the Creative Commons Attribution Non-Commercial License (http://creativecommons.org/licenses/by-nc/4.0/) which permits unrestricted non-commercial use, distribution, and reproduction in any medium, provided the original work is properly cited. ment of the lower limb is determined on standing, full-length, hip-to-ankle radiographs and is measured as the angle between the mechanical axis of the femur and the mechanical axis of the tibia. Intraoperatively, coronal alignment is determined using either long rods or a cautery wire during conventional TKA or by navigation during computer-assisted TKA.

Navigation has been reported to accurately measure mechanical alignment during $\mathrm{TKA}^{1-3)}$. Most surgeons who perform navigated TKA also obtain a preoperative standing, full-length, hip-to-ankle radiograph in their patients in order to measure the severity of knee deformity and degree of mechanical malalignment and plan the surgical procedure preoperatively. However, there may be a mismatch between the radiographic and navigation measurements of mechanical alignment for the same patient during $\mathrm{TKA}^{4)}$. This may be due to the non-weight bearing conditions during navigation when compared to weight bearing during fulllength radiography and the dynamic nature of knee deformities 
which may change with anaesthesia and muscle relaxation.

An associated sagittal deformity (either flexion or hyperextension) is frequently seen in arthritic knees undergoing TKA ${ }^{5,6)}$. However, the effect of sagittal knee deformity on coronal mechanical alignment measurements in patients undergoing TKA has not been previously studied. Hence the purpose of this study was to determine correlation between coronal mechanical alignment measured on preoperative standing full-length radiographs and navigation during TKA in the presence of an associated sagittal deformity (hyperextension and flexion deformity). We hypothesised that an associated sagittal knee deformity may lead to significant differences between radiographic and navigation measurements of mechanical alignment. To the best of our knowledge, this is the first study in the literature which has analysed the effect of sagittal knee deformity on coronal mechanical alignment measurements using preoperative radiographs and navigation during TKA.

\section{Materials and Methods}

A consecutive series of 212 knees which underwent computerassisted, primary TKA by a single surgeon between July 2015 and December 2015 were prospectively enrolled in this study. The inclusion criterion was all knees which underwent primary, computer-assisted TKA for tricompartmental knee arthritis. The exclusion criteria were cases where navigation had to be abandoned due to loosening of array pins during the course of surgery, improperly taken preoperative full-length, standing, hipto-ankle radiographs where limbs were in excessive rotation, and previous hip surgery.

Based on the exclusion criteria, 7 knees were excluded due to improperly taken radiographs, 4 knees were excluded due to loosening of array pins intraoperatively and 1 knee was excluded due to previous hip surgery. After exclusion, data from 200 primary, computer-assisted TKAs, performed in 149 patients (21 males and 128 females) was available for analysis. Of these, 98 patients had unilateral TKAs and 51 patients had bilateral TKAs. All TKAs were performed by a single surgeon using the imagefree Ci Navigation System (Brainlab AG, Munich, Germany). All knees were approached using an anterior longitudinal incision and a medial parapatellar arthrotomy, and a cemented, posterior cruciate-substituting design (PFC Sigma; DePuy Synthes, Warsaw, IN, USA) was used and the patella resurfaced in all patients.

Using two infra-red reflecting arrays fixed to the proximal tibia and distal femur, registration was performed in the standard fashion. The mechanical axis of the lower limb was obtained by navigation software using the center of the femoral head, the center of the intercondylar notch, centre of the tibial plateau and the center of the ankle plafond. After fixation of arrays and registration, intraoperative alignment (in both coronal and sagittal planes) was recorded by the operating surgeon with leg held in maximum extension and maximum varus or valgus as per the type of deformity, patella reduced and foot in neutral rotation. Intraoperative measurements of coronal and sagittal deformity was performed by one senior surgeon in all cases. Intraoperative measurement of coronal and sagittal deformities using navigation is reported to have high reliability and reproducibility ${ }^{7}$. Cutting blocks were navigated into position to perform the appropriate bone cuts where the tibial and femoral cuts were performed at $90^{\circ}$ relative to their mechanical axes in the coronal plane. The degree of soft tissue release was governed by the amount of soft tissue tightness assessed using a tensioning device and medial and lateral gap imbalance as quantified by navigation. Medial release for varus knees and lateral release for valgus knees were performed to achieve rectangular balanced gaps and a fully restored mechanical axis. The final alignment after cementing was also recorded by the operating surgeon using a method similar to the one described above.

All full-length radiographs were screened by one of the authors for excessive rotation of the limb which made the radiograph unsuitable for analysis. Excessive rotation of the limb was determined on radiographs by the profile of the lesser trochanter and fibular head and whether the patella was centred or medial/ lateral. Radiographs which showed excessive profile of the lesser trochanter or fibular head were considered to be in excessive rotation and excluded. Angles measured on full-length hip-toankle radiographs have been reported to be reliable and reproducible $^{8)}$. The degree of preoperative knee deformity or hipknee-ankle (HKA) angle was determined on the standing fulllength radiographs as the angle between the mechanical axis of the femur (center of the femoral head to the center of the knee) and the mechanical axis of the tibia (center of the tibial plateau to the center of the ankle plafond). All digital radiographic images were analyzed and measured by one orthopaedic surgeon from the team using Image image processing and analysis software ver. 1.41 (National Institutes of Health, Bethesda, MD, USA). The preoperative HKA angle as measured on full-length radiographs was compared with the initial mechanical coronal alignment as determined by navigation using the $t$-test. Knees were further subdivided into those having flexion deformity $\leq 10^{\circ}$, flexion deformity $>10^{\circ}$ and hyperextension and the coronal mechanical alignment was compared between the full-length radiograph and 
navigation groups using the $t$-test. A p-value of $<0.05$ was taken to be statistically significant. Pearson correlation coefficient was calculated to determine correlation between radiographic and navigation measurements of coronal mechanical alignment.

\section{Results}

The mean difference in coronal mechanical alignment measurements between full-length radiographs and navigation was significantly greater in knees with flexion deformity $>10^{\circ}$ versus flexion deformity $\leq 10^{\circ}$ ( $\left.\mathrm{p}=0.001\right)$ whereas it was not significant in knees with hyperextension versus flexion deformity $\leq 10^{\circ}$ $(\mathrm{p}=0.13)$ and in knees with hyperextension versus flexion deformity $>10^{\circ}(\mathrm{p}=0.28)$ (Table 1$)$. In knees with flexion deformity $>10^{\circ}, 48 \%$ of knees had a difference of $\geq 3^{\circ}$ with a range from $0.2^{\circ}$ to $14.7^{\circ}$ between the full-length radiograph and navigation alignment measurements which was significantly higher $(\mathrm{p}=0.04)$ when compared to $30 \%$ in knees with flexion deformity $\leq 10^{\circ}$.

Overall, there was no significant difference $(p=0.47)$ in the mean coronal mechanical alignment between the full-length radiograph and navigation measurements (Table 1). Similarly, there was no significant difference in the mean mechanical alignment between the full-length radiograph and navigation measurements in knees with hyperextension ( $\mathrm{p}=0.49)$, knees with flexion deformity $\leq 10^{\circ}(\mathrm{p}=0.45)$ and in knees with flexion deformity $>10^{\circ}(\mathrm{p}=0.32)$.

Overall, the mean coronal mechanical alignment measured on full-length radiographs showed a strong positive correlation with the navigation measurements $(\mathrm{r}=0.88, \mathrm{p}<0.001)$. Similarly, mean mechanical alignment measurements showed a strong positive correlation in knees with hyperextension $(r=0.89, \mathrm{p}<0.001)$ and knees with flexion deformity $\leq 10^{\circ}(\mathrm{r}=0.84, \mathrm{p}<0.001)$, and mod- erate positive correlation in knees with flexion deformity $>10^{\circ}$ $(\mathrm{r}=0.74, \mathrm{p}<0.001)$.

\section{Discussion}

The findings of this study suggest that there was no overall difference in the mean coronal mechanical alignment between the full-length radiograph and navigation measurements and there was a strong correlation between the two techniques. However, the mean difference in mechanical alignment between the two techniques was significantly greater $(\mathrm{p}=0.001)$ in patients with associated flexion deformity $>10^{\circ}$ when compared to knees with associated flexion deformity $\leq 10^{\circ}$, and $48 \%$ of knees with a flexion deformity $>10^{\circ}$ had a difference of $\geq 3^{\circ}$ (ranging from $0.2^{\circ}$ to $\left.14.7^{\circ}\right)$ between the full-length radiograph and navigation measurements (Fig. 1).

Babazadeh et al. ${ }^{9)}$ in a prospective analysis of 40 knees reported a strong correlation $(\mathrm{r}=0.78)$ between navigation and radiographic coronal mechanical alignment measurements which was similar to the findings from our study. Willcox et al. ${ }^{4)}$ in a retrospective review of 185 patients reported a mean difference of $-0.4^{\circ}$ between preoperative radiographic and pre-implantation navigation measurements for coronal mechanical alignment with an intra-class correlation coefficient of 0.90 . In contrast, the mean difference in coronal mechanical alignment between the two measurement techniques was much higher in the current study at $2.6^{\circ}$. This could be due to the fact that 44 knees $(22 \%)$ in the current study had an associated flexion deformity of $>10^{\circ}$ and 30 knees (15\%) had an associated hyperextension deformity.

There may be disagreement between navigation and radiographic mechanical alignment measurements due to their individual limitations. Computer navigation determines mechanical

Table 1. Comparison of Mechanical Alignment on Full-Length Radiographs vs. Navigation According to the Type of Sagittal Deformity

\begin{tabular}{lcccc}
\hline \multicolumn{1}{c}{ Parameter } & All knees & Hyperextension & $\begin{array}{c}\text { Fixed flexion } \\
\text { deformity } \leq 10^{\circ}\end{array}$ & $\begin{array}{c}\text { Fixed flexion } \\
\text { deformity }>10^{\circ}\end{array}$ \\
\hline $\begin{array}{l}\text { No. of knees } \\
\text { Sagittal deformity }\left(^{\circ}\right)\end{array}$ & 200 & 30 & 126 & 44 \\
$\begin{array}{l}\text { Mechanical alignment on full-length } \\
\text { radiograph }\left(^{\circ}\right)\end{array}$ & $10.2 \pm 6.9(-25.2$ to 27.6$)$ & $11.5 \pm 8.9(-10.3$ to 27.6$)$ & $10.5 \pm 5.4(-12.8$ to 26.2$)$ & $13 \pm 8.4(-25.2$ to 27$)$ \\
$\begin{array}{l}\text { Mechanical alignment on navigation }\left(^{\circ}\right) \\
\text { Difference in mechanical alignment }\left(^{\circ}\right)\end{array}$ & $10.7 \pm 7(-30.5$ to 28$)$ & $10.1 \pm 8.2(-11.5$ to 22$)$ & $11 \pm 5.1(-10$ to 26$)$ & $14.8 \pm 8.7(-30.5$ to 28$)$ \\
$\begin{array}{l}\text { Limbs with mechanical alignment } \\
\text { difference of } \geq 3^{\circ}(\%)\end{array}$ & $35.5(71 / 200)$ & $2.7 \pm 1.8(0.5$ to 7.8$)$ & $2.2 \pm 1.6(0$ to 7.2$)$ & $3.4 \pm 3.2(0.2$ to 14.7$)$ \\
\hline
\end{tabular}

Values are presented as mean \pm standard deviation (range or number). Negative values for sagittal deformity denote hyperextension and positive values denote flexion. Negative values for limb mechanical alignment denote valgus and positive values denote varus. 


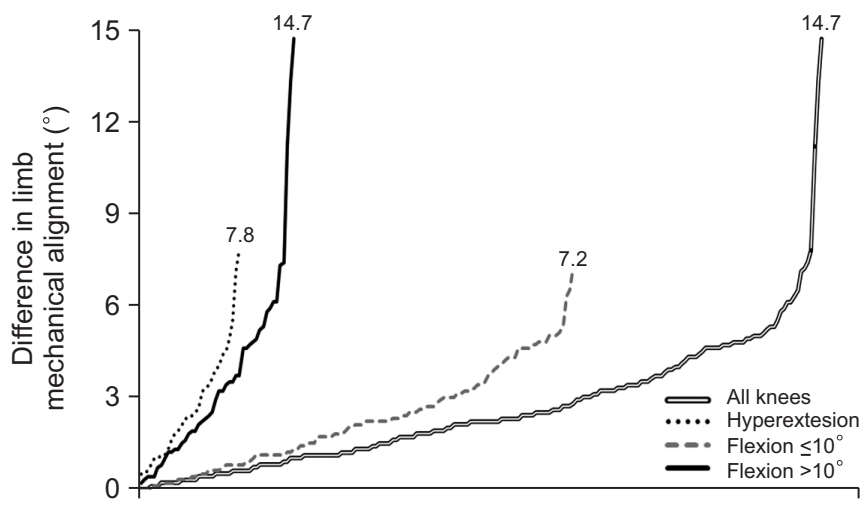

No. of knees

Fig. 1. Distribution of knees according to the difference in coronal mechanical alignment between the radiographic and navigation measurements in different types of sagittal deformity.

alignment based on anatomical landmarks registered during surgery, and improper registration may lead to an error in calculating the mechanical alignment by the navigation software. Additionally, measurements taken during navigation are made under non-weight bearing conditions (albeit with maximum force applied by the surgeon in the direction of the deformity to mimic weight bearing) after arthrotomy and preliminary exposure of the knee joint has been performed. Furthermore, variations in how the limb is held (excessive rotation) while measuring mechanical alignment with navigation may also add to measurement errors.

Mechanical coronal alignment measurements on preoperative full-length radiographs may be affected by malposition of the lower limbs during radiography. Although previous studies have reported correlation between radiographic and navigation measurements of coronal mechanical alignment in patients undergoing $\mathrm{TKA}^{4,9)}$, the factors which may cause differences in these two measurement techniques are still unclear. Dexel et al. ${ }^{10)}$ in a retrospective analysis of 135 patients reported that although the severity of knee deformity in the coronal plane may not lead to large differences, preoperative radiographs taken with limbs in excessive malrotation can cause large differences in coronal mechanical alignment measurements when the two techniques were compared. The findings of the current study suggest that an associated flexion deformity $>10^{\circ}$ may lead to significant discrepancy between radiographic and navigation measurements of mechanical alignment. However, this discrepancy was not significant when the knee had an associated hyperextension or flexion deformity $\leq 10^{\circ}$. Hence, computer navigation may be more reliable than full-length radiographs for determining coronal mechanical alignment in knees with significant flexion deformity during TKA.
In the authors' opinion, varus arthritic knees undergoing TKA with an associated flexion deformity $>10^{\circ}$ have significant contracture of the posteromedial soft-tissue structures whereas in knees with flexion deformity $\leq 10^{\circ}$ this contracture is not severe and in knees with hyperextension the posterior soft-tissues are $\operatorname{lax}^{11)}$. It is our conjecture that this asymmetric soft-tissue contracture in arthritic knees with severe flexion deformity is probably the reason for significant discrepancy between radiographic and navigation measurements of coronal mechanical alignment in knees with flexion deformity $>10^{\circ}$ when compared to knees with hyperextension or flexion deformity $\leq 10^{\circ}$. However, we had only one knee with an associated hyperextension deformity of $>10^{\circ}$ and the effect of significant hyperextension on radiographic measurements is unclear.

The current study had some limitations. Both preoperative radiographic and navigation measurements were performed by single (different) individuals, which has the risk of measurement errors. However, both these techniques have been reported to be reliable and reproducible with good intra- and inter-observer correlation ${ }^{7,8)}$. The current study used a single infrared-based imageless navigation system to measure coronal mechanical alignment. Although reliable and reproducible, alignment measurements can vary between imageless and computed tomographybased navigation systems ${ }^{12)}$ and hence the findings of the current study need to be validated using different navigation systems. To the best of our knowledge, this is the first study in the literature which has analysed the effect of sagittal knee deformity on coronal mechanical alignment measurements using preoperative radiographs and navigation during TKA.

\section{Conclusions}

Despite the strong correlation between the full-length radiograph and navigation techniques for mean coronal mechanical alignment, the mean difference in mechanical alignment between the two techniques was significantly higher when the patient had an associated flexion deformity $>10^{\circ}$. Hence, surgeons should take caution when relying on preoperative full-length radiographs for determining coronal mechanical alignment in patients with an associated flexion deformity $>10^{\circ}$ where using navigation may be more reliable.

\section{Conflict of Interest}

No potential conflict of interest relevant to this article was reported. 


\section{References}

1. Cheng T, Zhao S, Peng X, Zhang X. Does computer-assisted surgery improve postoperative leg alignment and implant positioning following total knee arthroplasty? A metaanalysis of randomized controlled trials? Knee Surg Sports Traumatol Arthrosc. 2012;20:1307-22.

2. Mason JB, Fehring TK, Estok R, Banel D, Fahrbach K. Metaanalysis of alignment outcomes in computer-assisted total knee arthroplasty surgery. J Arthroplasty. 2007;22:1097-106.

3. Mullaji A, Kanna R, Marawar S, Kohli A, Sharma A. Comparison of limb and component alignment using computerassisted navigation versus image intensifier-guided conventional total knee arthroplasty: a prospective, randomized, single-surgeon study of 467 knees. J Arthroplasty. 2007;22: 953-9.

4. Willcox NM, Clarke JV, Smith BR, Deakin AH, Deep K. A comparison of radiological and computer navigation measurements of lower limb coronal alignment before and after total knee replacement. J Bone Joint Surg Br. 2012;94:123440.

5. Su EP. Fixed flexion deformity and total knee arthroplasty. J Bone Joint Surg Br. 2012;94(11 Suppl A):112-5.

6. Baldini A, Castellani L, Traverso F, Balatri A, Balato G, Franceschini V. The difficult primary total knee arthroplasty: a review. Bone Joint J. 2015;97(10 Suppl A):30-9.

7. Jenny JY, Boeri C, Picard F, Leitner F. Reproducibility of intra-operative measurement of the mechanical axes of the lower limb during total knee replacement with a non-imagebased navigation system. Comput Aided Surg. 2004;9:161-5.

8. Skytta ET, Haapamaki V, Koivikko M, Huhtala H, Remes V. Reliability of the hip-to-ankle radiograph in determining the knee and implant alignment after total knee arthroplasty. Acta Orthop Belg. 2011;77:329-35.

9. Babazadeh S, Dowsey MM, Bingham RJ, Ek ET, Stoney JD, Choong PF. The long leg radiograph is a reliable method of assessing alignment when compared to computer-assisted navigation and computer tomography. Knee. 2013;20:242-9.

10. Dexel J, Kirschner S, Gunther KP, Lützner J. Agreement between radiological and computer navigation measurement of lower limb alignment. Knee Surg Sports Traumatol Arthrosc. 2014;22:2721-7.

11. Mullaji A, Lingaraju AP, Shetty GM. Computer-assisted total knee replacement in patients with arthritis and a recurvatum deformity. J Bone Joint Surg Br. 2012;94:642-7.

12. Carli A, Aoude A, Reuven A, Matache B, Antoniou J, Zukor DJ. Inconsistencies between navigation data and radiographs in total knee arthroplasty are system-dependent and affect coronal alignment. Can J Surg. 2014;57:305-13. 